\title{
Punctured two-dimensional sheets for harvesting
}

\section{blue energy}

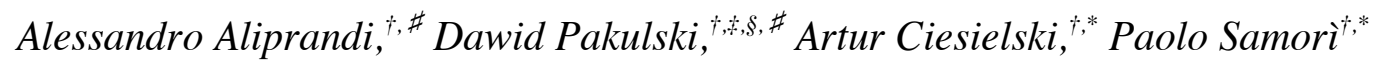

${ }^{\dagger}$ Université de Strasbourg, CNRS, ISIS, 8 alleé Gaspard Monge, 67000 Strasbourg, France

${ }^{*}$ Faculty of Chemistry, Adam Mickiewicz University, Umultowska 89b, 61-614 Poznań, Poland.

${ }^{\S}$ Centre for Advanced Technologies, Adam Mickiewicz University, Umultowska 89c, 61614

Poznań, Poland.

\# These two authors contributed equally to this work.

KEYWORDS. Two-dimensional materials, membranes, blue energy. 
ABSTRACT The challenges of the global climate change and the World's progressively growing demand for energy have brought the need for new renewable energy sources to the top of the international community's agenda. It is well known since many centuries that energy is released upon mixing seawater and freshwater, yet only a few decades ago it became clear how this energy can be converted into electricity instead of heat. As a result, the blue energy rush has raised and set new strategies in different science and technology sectors leading to construction of new generation of plants and other technological investments. Among many approaches, pressure retarded osmosis has emerged as promising method to collect the largest amount of produced blue energy. In this Perspective, we highlight the advances in the development of ultrathin membranes based on two-dimensional materials. We discuss the most relevant synthetic methods devised to generate atomically thin membranes for pressure-retarded osmosis and retarded electrodialysis applications, and we provide some critical views on the greatest challenges in this thrilling research area.

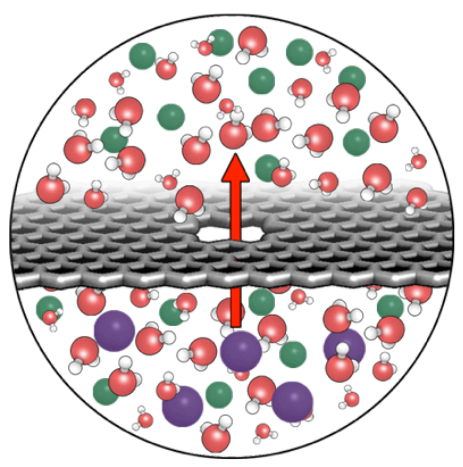

Punctured two-dimensional sheets for harvesting blue energy 
One among the greatest problems our society has to face, in view of the continuous growth of population and climate changes, is the scarcity of fresh water, which amounts to only $2.5 \%$ of the World's water while the remaining $97.5 \%$ consists of salty water. ${ }^{1}$ This forces the scientists to urgently find new approaches for the desalination of seawater on an industrial scale. Even though the state-of-the-art desalination technologies are based since long time on membranes operating via the reverse osmosis (RO) process, ${ }^{2}$ only a few decades ago we have learnt how such membranes can also be also employed to convert the energy released by salinity gradient into electricity, also known as blue energy or osmotic power (OP). The mechanism of the process relies on naturally occurring osmosis, where the (solvent) molecules pass through a semipermeable membrane from a dilute solution into a more concentrated solution mainly by means of two process, i.e. pressure-retarded osmosis (PRO) and reverse electrodialysis (RED). In PRO the osmotic pressure difference drives water across a salt-rejecting semipermeable membrane into a more concentrated solution. The resulting volume expansion in the latter can be converted into energy by depressurization through a hydroturbine. Conversely, RED process is driven by the Nernst potential, thus the salinity gradient is directly converted into electricity. This is obtained by using ion exchange membrane pairs, whose selectively allows for counterion permeation, resulting into a net ion flux (Figure 1). PRO and RED have fundamentally different working principles as well as operating constraints. For example the membranes used in PRO have to withstand tremendous hydraulic pressure (water flux in the order of $\left.20-50 \mathrm{~L} \mathrm{~m}^{-2} \mathrm{~h}^{-1}\right)^{3}$ whereas the mechanical strength of the membrane in the RED system is not as crucial as the ionic resistance and permselectivity. ${ }^{4}$ 
a)

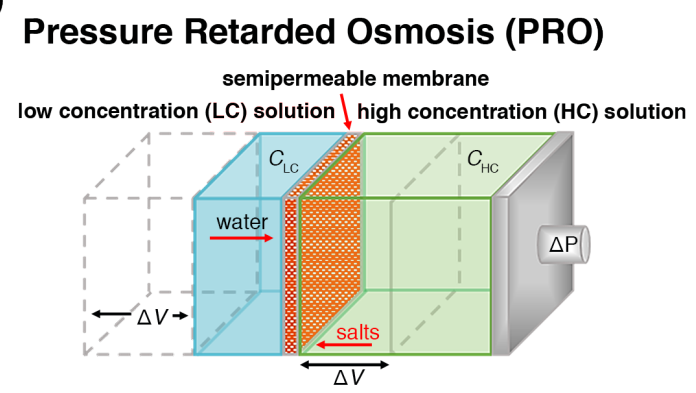

b)

Reverse Electrodialysis (RED)

cation exchenge membrane (CEM) anion exchenge membrane (AEM)

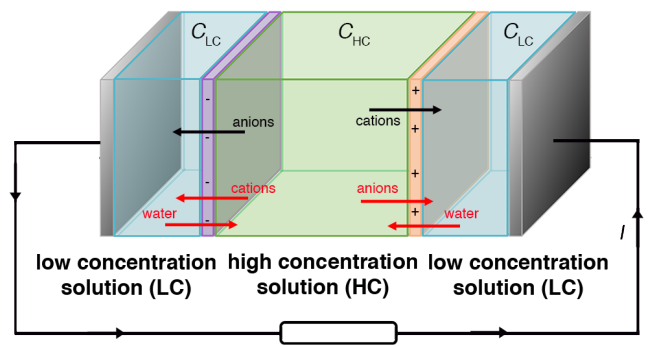

Figure 1. Schematic representation of (a) pressure retarded osmosis (PRO), and (b) reverse electrodialysis (RED). In PRO, osmotic driving force for water flux across the semipermeable membrane is produced through the salinity gradient, and the increasing volume of the pressurized high concentration (HC) solution powers a hydroturbine. In RED, the concentration difference across the ion exchange membranes produces a Nernst potential and the membranes selectively allow the transport of counterions. The ion flux is converted into an electric current (I). In both devices, i.e. PRO and RED, some salt leaks from the HC solution to the low concentration (LC) solution as the membranes are not perfectly selective.

Since water transport through a membrane scales inversely with the membrane thickness, ${ }^{5}$ recently, the use of atomically thick materials such as graphene and other two-dimensional materials (2DMs) have been considered as an alternative approach towards OP membranes, since the atomic thickness can minimize the transport resistance and maximize the flux. ${ }^{1,6}$ Thus a control over the membrane thickness down to a single atomic layer and the ability to introduce controlled, subnanometer-sized pores at high density over large areas is crucial for their optimal integration in osmotic power nanogenerators. Indeed, the atomic thickness, high mechanical strength, and chemical inertness combined with intrinsic or induced porous structures generated via perforation or controllable assembly allow highly selective transport of different species (e.g. 
liquids, gases and ions) across such "punctured" two-dimensional sheets. ${ }^{6,7}$ The developments in the field of 2DMs, and in particular of graphene and layered transition-metal dichalcogenides (TMDCs), have opened a new era in the field of membrane separation. For example, recently, a single-layer molybdenum disulfide $\left(\mathrm{MoS}_{2}\right)$ with a single nanopore has been used to harvest $\mathrm{OP}{ }^{8}$ which can be used for powering a $\mathrm{MoS}_{2}$ transistor. Based on the membrane performance, it was extrapolated that power density reaches $10^{6} \mathrm{~W} \mathrm{~m}^{-2}$, being a million times grater than the power density obtained with classical exchange membranes (e.g. cellulose acetate or polyamide membranes) by RED, ${ }^{9}$ and nearly riches the values estimated theoretically for RED of sea water $\left(2.7 \times 10^{6} \mathrm{~W}\right){ }^{3}$

In this Perspective we discuss recent and most enlightening advancements in the preparation of atomically thin membranes based on graphene and $\mathrm{MoS}_{2}$ for power generation by reverse electrodialysis (RED) and pressure-retarded osmosis (PRO). In particular, we focus on the methods for the introduction of nanopores and the resulting chemical functionalization of 2DM's surface and pore edges. We will also discuss the performance of the resulting membranes for RED applications, and we will conclude by highlighting the major challenges and opportunities in the field of membranes for harvesting the blue energy.

Graphene In the case of graphene synthetized via chemical vapor deposition (CVD), isolated pores with a diameter smaller than $2 \mathrm{~nm}$ (Figure 2) can be obtained by making use of the focused electron beam of a transmission electron microscope (TEM) ${ }^{10-12}$ However, such an approach is not upscalable and time consuming; therefore, it cannot be used to generate multiple pores over large areas, which is compulsory for practical applications. In parallel, other approaches mainly based on oxidative processes, ${ }^{13-16}$ are being explored. However, the oxidative process has some 
major drawbacks. In particular, a general lack over the control of the pore size and size distribution has been reported, and it has been attributed to grain boundaries that are more reactive than the basal plane of graphene sheets.

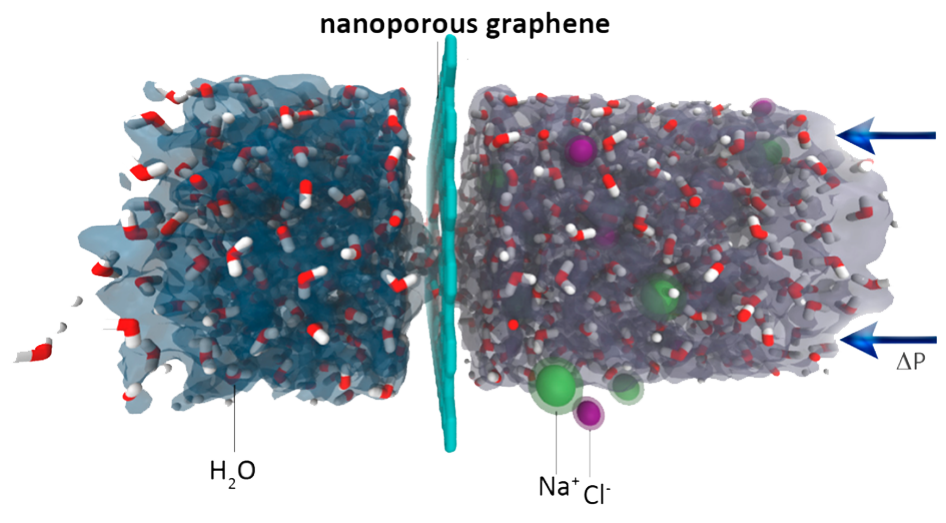

Figure 2. Graphical representation of PRO process exploiting nonporous graphene sheet as a membrane. Reproduced from Ref. ${ }^{7}$ with permission from American Chemical Society.

A possible solution to overcome this problem has been proposed by Russo et al. ${ }^{17}$ In order to take advantage of the difference in reactivity of CVD graphene sheets, a two-step process has been pursued. Firstly, defects are uniformly generated on the surface by argon ion bombardment and diffuse electron beam is used to selectively etch the edges. Such approach has been further developed by O'Hern et al. ${ }^{18}$ to create controlled, high-density, pores with sub-nanometer diameter over macroscopic areas of single-layer graphene flakes. Isolated defects are introduced onto graphene through ion bombardment with Gallium ions and then the defects are transformed into permeable pores using oxidative etching in acidic potassium permanganate. The defects introduced into the graphene lattice by the ion bombardment process can be monitored by Raman spectroscopy while the formation of nanopores can be observed using scanning transmission electron microscopy. Interestingly, in the absence of ion bombardment no pores have been 
observed during the course of the etching process; also Raman spectra remained relatively unaltered compared to pristine graphene. Such findings provide evidence that the nucleation of defects is a fundamental step towards the creation of the pores. Furthermore, after long etching time, the pore density approached the theoretically predicted defect density expected from the ion bombardment, which further corroborate the hypothesis that the observed pores results from enlargement of defects introduced by ion bombardment. Although the pore density scales with the etching time, the mean pore diameter increased significantly only during the first 30 min and then it appeared to stabilize at around $0.40 \mathrm{~nm}$ after $60 \mathrm{~min}$ etching time. Such trend suggests the existence of a slow, stochastic initiation of the reaction at the nucleated defect, which is followed by rapid growth until the pore stabilization. The authors have attributed this behavior to the functionalization of the pore edges by oxygen functional groups such as ketone, quinone, hydroxyl, or carboxyl groups, whose presence has been confirmed by X-ray photoelectron spectroscopy. Transport measurements across the created pores revealed that before the etching some transport of $\mathrm{KCl}$ as well as a larger organic dyes takes place even in the untreated graphene membrane, a behavior which has been ascribed to the presence of tears and defects. However, no ion selectivity has been observed between $\mathrm{K}^{+}$and $\mathrm{Cl}^{-}$species. Interestingly, upon etching, both the membrane potential and the rate of $\mathrm{KCl}$ diffusion increased thereby indicating the emergence of a modest selectivity in the transport of the $\mathrm{K}^{+}$over $\mathrm{Cl}^{-}$. Such feature has been ascribed to the negatively charged oxygen functionalities at the pore edges. However, the selectivity is lost upon increasing of the etching time, suggesting that supramolecular forces, and in particular electrostatic interactions, govern the ion selectivity only when the pores are small whereas steric effects dominate the transport through larger pores. 
A significant step forward in the preparation of graphene nanopores for reverse osmosis process has been reported by Mahurin et al. ${ }^{19}$ In this work a single-layer CVD graphene has been transferred onto silicon nitride $(\mathrm{SiN})$ microchip device with a single hole possessing a diameter of $5 \mu \mathrm{m}$, generated through the exposure to oxygen plasma. Raman spectroscopy has been shown to be a powerful tool to follow the formation of the nanopores since the intensity of the D peak, i.e. the characteristic peak revealing defects in the graphene, grows with increasing exposure time, thus the $I_{D} / I_{G}$ ratio can be conveniently used to follow the formation of the nanopores. While the pristine graphene (with $\mathrm{I}_{\mathrm{D}} / \mathrm{I}_{\mathrm{G}}=0$ ) is impermeable, the plasma-etched graphene samples $\left(\mathrm{I}_{\mathrm{D}} / \mathrm{I}_{\mathrm{G}} \leq 0.5\right)$ displayed almost $100 \%$ salt rejection in presence of dissolved monovalent ions $\left(\mathrm{K}^{+}\right.$, $\left.\mathrm{Na}^{+}, \mathrm{Li}^{+}, \mathrm{Cl}^{-}\right)$. Nevertheless, while no ionic current was detected, a measurable water transport was observed, indicating the presence of extremely small pores. Interestingly, in graphene exhibiting $\mathrm{I}_{\mathrm{D}} / \mathrm{I}_{\mathrm{G}}$ ratio exceeding 1 , both water transport and ionic current increase, demonstrating that extended plasma exposure not only enhances the number of structural defects but also enlarges the pores. The pore diameters for the membrane with $\mathrm{I}_{\mathrm{D}} / \mathrm{I}_{\mathrm{G}} \approx 1$ are in the range of 0.5 to $1.0 \mathrm{~nm}$, with pore density being on the order of 1 pore $/ 100 \mathrm{~nm}^{2}$. When the graphene membrane on the $\mathrm{SiN}$ microchip device separates two liquids, i.e. $1 \mathrm{M} \mathrm{KCl}$ solution and deionized water, a net osmotic pressure difference between the two compartments raises. This feature can be clearly observed by a change in the water level difference between the capillaries connected to the opposite water chambers, unambiguously confirming the semipermeable nature of the plasmatreated graphene membranes and their possible application in PRO. Noteworthy, Raman spectroscopy does not provide any information on the chemical nature of the defects, and therefore the mechanism of the formation of such nanopores is still poorly understood. 
Golovchenko et al..$^{20,21}$ have formed freestanding nano-punctured suspended graphene sheets through electrical treatment of graphene in electrolyte solution. In particular, the authors have used $7 \mathrm{~V}$ pulses $(250 \mathrm{~ns})$ to induce the nucleation of a nanopores, which has been followed by lower voltage pulses of $5 \mathrm{~V}(250 \mathrm{~ns})$ to controllably increase the pore size. Interestingly, such nano-punctured graphene preferentially permit the transport of $\mathrm{K}^{+}$over $\mathrm{Cl}^{-}$, with selectivity ratio exceeding 100, and conduct monovalent cations up to 5 times more rapidly than divalent cations. The observed $\mathrm{K}^{+} / \mathrm{Cl}^{-}$selectivity is retained even in pores as large as $20 \mathrm{~nm}$ in diameter, which is apparently in contrast with the trend previously reported by O'Hern ${ }^{18}$ on graphene membranes with multiple subnanometer pores. Nonetheless, the $\mathrm{pH}$ dependence of cation/anion selectivity, which persists in $20 \mathrm{~nm}$ pores, suggests that the deprotonatable oxygen-containing groups are located not only at the pore edges but also on the plane of graphene sheets. Indeed, the presence of a negatively charged surface is expected to enhance the screening of positive counterions, as well as repelling anions in solution causing the total ionic current to be cation-selective. These results suggest that high throughput, highly selective graphene RED membranes can be fabricated without the need for subnanometer control over pore size, if surface charges are also brought into play.

Molybdenum disulfide When compared to graphene, a $\mathrm{MoS}_{2}$ layer comprises two types of atoms, thus is in theory it allows to craft the pore edge with either Mo or S atoms or mixture of both, which can result in different functionality of nano-punctured sheets. Theoretical calculations ${ }^{22}$ have shown that $\mathrm{MoS}_{2}$ membranes are promising material for water purification and salt rejection. In particular, among all possible $\mathrm{MoS}_{2}$ pore architectures, pure Mo pores are predicted to perform the best. Modeling suggests that pore areas ranging from 20-60 $\AA^{2}$ should 
be suitable to reject overs $88 \%$ of salts even at theoretically high pressures of $350 \mathrm{MPa}$. Moreover, water permeation rates are expected to be $70 \%$ higher than the nano-punctured graphene, since water molecules can slip over the hydrophobic edges of $S$, being attracted by the hydrophilic sites of Mo at the pore center. This arrangement of hydrophobic and hydrophilic atoms along with the conical shape of the pore is indeed expected to enhance the flux of water. Recently, Radenovic et al., ${ }^{23}$ have shown that, similarly to graphene, nanopores of variable size can be generated in CVD $\mathrm{MoS}_{2}$ sheets by making use of focused electron beam of TEM. Since such process is time-consuming, expensive and not scalable, electrochemical approaches need to be exploited by taking particular advantage of the electrochemical activity of $\mathrm{MoS}_{2} \cdot{ }^{24}$ The monitoring of the step-like feature formation in the ionic current through the nanopore formation provides a direct feedback on the nanopore size. Such steps consist in a first introduction of defects or single atom vacancy, followed by the successive removal of individual atoms or unit cells from the single-layer $\mathrm{MoS}_{2}$ lattice. The application of a single layer $\mathrm{MoS}_{2}$ nanopores for RED application has been tested by the same group (Figure 3 ). ${ }^{8}$ The osmotic power is generated by separating two reservoirs containing $\mathrm{KCl}$ solutions of different concentrations with a freestanding $\mathrm{MoS}_{2}$ sheet with a single nanopore of diameter ranging between 2 to $25 \mathrm{~nm}$. The highly negatively charged surface allows for selective $\mathrm{K}^{+}$ions flow, resulting into a net osmotic current. The performance of the system has been characterized in terms of ionic transport properties as a function of both salt concentration and $\mathrm{pH}$. Indeed the large pore conductance, which originates from the atomically thin nature of the membrane, is also highly dependent on both salt concentration and $\mathrm{pH}$ pointing toward the presence of surface charge on the pore. Interestingly, the increase in $\mathrm{pH}$ leads to higher generated voltages and currents, suggesting the importance of surface charges on the ion-selective process and consequently affecting the 
osmotic energy conversion. Likewise, the size of the pore plays an important in the process: small pores display better voltage behavior reflecting improved performance in terms of ion selectivity which decreases with the increasing pore size. The power density of a single-layer $\mathrm{MoS}_{2}$ membrane with a homogeneous pore size of $10 \mathrm{~nm}$ has been estimated as high as $10^{6} \mathrm{~W}$ $\mathrm{m}^{-2}$. Remarkably, this value is a million times higher than the power density obtained by RED with classical exchange membranes.

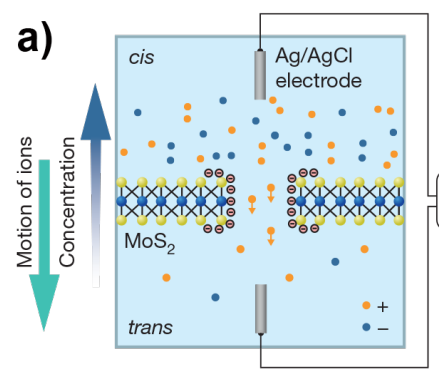

b)

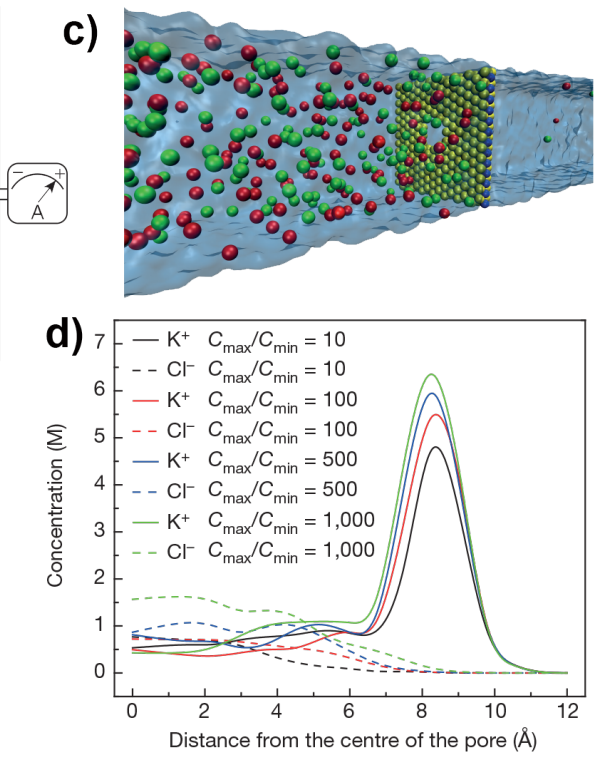

Figure 3. a) Schematic representation of the experimental set-up for harvesting osmotic energy with $\mathrm{MoS}_{2}$ nanopores; b) Example of a TEM-drilled $\mathrm{MoS}_{2}$ nanopore of diameter $5 \mathrm{~nm}$; c) Top panel, a typical simulation box used in molecular-dynamics simulations, showing the nanopore membrane (in blue and yellow) and the salt (green and red) in solution; c) Molecular-dynamicssimulated potassium-ion and chloride-ion concentrations as a function of the radial distance from the center of the pore. The region near the charged wall of the pore is representative of the electrical double layer. $\mathrm{C}_{\max }$, maximum concentration; $\mathrm{C}_{\min }$, minimum concentration. Reproduced from Ref. ${ }^{8}$ with permission from Nature Publishing Group. 
The consistency between experimental results and theoretical models highlights three important factors in achieving efficient power generation from a nano-punctured single-layer $\mathrm{MoS}_{2}$, i.e. the size of the pores, the thickness of the membrane and the surface charge.

\section{CONCLUSIONS AND OUTLOOKS}

Due to their unique physico-chemical properties, two-dimensional materials (2DMs) hold tremendous potential for harvesting blue energy. Nevertheless, the development of atomically thin membranes based on 2DMs for PRO and RED applications remains challenging as the controlled chemical functionalization of both surface and pore edges is crucial to obtain permselective membranes. Among various approaches, the defect engineering via accurate perforation by making use of electrochemical methods appears to be the most promising in terms of the low complexity of production and scaling up towards the mass production. We believe that electrochemical functionalization can be combined with the electrochemical perforation to tune the surface charges and pore edge functionalities, thus improving the performance of the membrane keeping possible the mass scale production. The possibility to power a low voltage transistor by harvesting osmotic power has been demonstrated thus parallelization can be already exploited. We can foresee challenges and opportunities to foster new generations of PRO and RED membranes through chemical engineering of the pores or even the 2DMs themselves. The latter, also known as 2D covalent organic frameworks (COFs), could allow for fine-tuning the pore sizes and their density, as well as the selectivity of the membrane toward specific chemical species. Yet, their mechanical stability will decrease with the increasing number of pores and therefore the use of additional substrates will be required to increase the resistance towards the water flux. Moreover, alongside the CVD methods, liquid-phase exfoliation (LPE) methods are 
becoming more and more interesting because they are extremely versatile, up-scalable, and can be used to deposit 2DMs in a variety of environments and on different substrates not available using CVD growth methods. Despite the recent advances discussed in this Perspective the translation of the developed knowledge into practical and large scale application are far from being achieved, yet, it can be expected to have significant impact in future developments in a field at the cross-roads between nanotechnology and materials science, with the ultimate goal of establishing a real technology for the harvesting of blue energy.

\section{AUTHOR INFORMATION}

\section{Corresponding Author}

A.C.ciesielski@unistra.fr, P.S. samori@unistra.fr

\section{Author Contributions}

The manuscript was written through contributions of all authors. All authors have given approval to the final version of the manuscript.

\section{Conflict of Interest}

The authors declare no competing financial interest.

\section{ACKNOWLEDGMENT}

This work was supported by the European Commission through the Graphene Flagship Core 1 project (GA-696656) and the Polish National Science Centre (Grant no. 2015/18/E/ST5/00188). We also are thankful for the support of the Agence Nationale de la Recherche through the LabEx project Chemistry of Complex Systems (ANR-10-LABX-0026_CSC), the International Center 
for Frontier Research in Chemistry (icFRC). D.P. acknowledges the support from the Embassy of France in Poland in the form of a cotutelle doctoral program at the University of Strasbourg.

\section{REFERENCES}

1. Dervin, S.; Dionysiou, D. D.; Pillai, S. C. 2D Nanostructures for Water Purification: Graphene and Beyond. Nanoscale 2016, 8, 15115-15131.

2. Shannon, M. A.; Bohn, P. W.; Elimelech, M.; Georgiadis, J. G.; Marinas, B. J.; Mayes, A. M. Science and Technology for Water Purification in the Coming Decades. Nature 2008, 452, 301-310.

3. Helfer, F.; Lemckert, C.; Anissimov, Y. G. Osmotic Power with Pressure Retarded Osmosis: Theory, Performance and Trends - A Review. J. Membr. Sci. 2014, 453, 337-358.

4. Hong, J. G.; Zhang, B.; Glabman, S.; Uzal, N.; Dou, X.; Zhang, H.; Wei, X.; Chen, Y. Potential Ion Exchange Membranes and System Performance in Reverse Electrodialysis for Power Generation: A Review. J. Membr. Sci. 2015, 486, 71-88.

5. Suk, M. E.; Aluru, N. R. Water Transport through Ultrathin Graphene. J. Phys. Chem. Lett. 2010, 1, 1590-1594.

6. Zheng, Z.; Grünker, R.; Feng, X. Synthetic Two-Dimensional Materials: A New Paradigm of Membranes for Ultimate Separation. Adv. Mater. 2016, 28, 6529-6545.

7. Cohen-Tanugi, D.; Grossman, J. C. Water Desalination Across Nanoporous Graphene. Nano Lett. 2012, 12, 3602-3608. 
8. Feng, J.; Graf, M.; Liu, K.; Ovchinnikov, D.; Dumcenco, D.; Heiranian, M.; Nandigana, V.; Aluru, N. R.; Kis, A.; Radenovic, A. Single-layer MoS2 Nanopores as Nanopower Generators. Nature 2016, 536, 197-200.

9. Logan, B. E.; Elimelech, M. Membrane-Based Processes for Sustainable Power Generation Using Water. Nature 2012, 488, 313-319.

10. Garaj, S.; Hubbard, W.; Reina, A.; Kong, J.; Branton, D.; Golovchenko, J. A. Graphene as a Subnanometre Trans-Electrode Membrane. Nature 2010, 467, 190-193.

11. Garaj, S.; Liu, S.; Golovchenko, J. A.; Branton, D. Molecule-Hugging Graphene Nanopores. Proc. Natl. Acad. Sci. U. S. A. 2013, 110, 12192-12196.

12. Merchant, C. A.; Healy, K.; Wanunu, M.; Ray, V.; Peterman, N.; Bartel, J.; Fischbein, M. D.; Venta, K.; Luo, Z.; Johnson, A. T. C.; Drndić, M. DNA Translocation through Graphene Nanopores. Nano Lett. 2010, 10, 2915-2921.

13. Koenig, S. P.; Wang, L.; Pellegrino, J.; Bunch, J. S. Selective Molecular Sieving Through Porous Graphene. Nat. Nano. 2012, 7, 728-732.

14. Bai, J.; Zhong, X.; Jiang, S.; Huang, Y .; Duan, X. Graphene Nanomesh. Nat. Nano. 2010, $5,190-194$.

15. Cheng, Y. C.; Kaloni, T. P.; Zhu, Z. Y.; Schwingenschlögl, U. Oxidation of Graphene in Ozone Under Ultraviolet Light. Appl. Phys. Lett. 2012, 101, 073110. 
16. Liu, L.; Ryu, S.; Tomasik, M. R.; Stolyarova, E.; Jung, N.; Hybertsen, M. S.; Steigerwald, M. L.; Brus, L. E.; Flynn, G. W. Graphene Oxidation: Thickness-Dependent Etching and Strong Chemical Doping. Nano Lett. 2008, 8, 1965-1970.

17. Russo, C. J.; Golovchenko, J. A. Atom-by-atom Nucleation and Growth of Graphene Nanopores. Proc. Natl. Acad. Sci. U. S. A. 2012, 109, 5953-5957.

18. O’Hern, S. C.; Boutilier, M. S. H.; Idrobo, J.-C.; Song, Y.; Kong, J.; Laoui, T.; Atieh, M.; Karnik, R. Selective Ionic Transport through Tunable Subnanometer Pores in Single-Layer Graphene Membranes. Nano Lett. 2014, 14, 1234-1241.

19. Surwade, S. P.; Smirnov, S. N.; Vlassiouk, I. V.; Unocic, R. R.; Veith, G. M.; Dai, S.; Mahurin, S. M. Water Desalination Using Nanoporous Single-Layer Graphene. Nat. Nano. 2015, $10,459-464$.

20. Kuan, A. T.; Lu, B.; Xie, P.; Szalay, T.; Golovchenko, J. A. Electrical Pulse Fabrication of Graphene Nanopores in Electrolyte Solution. Appl. Phys. Lett. 2015, 106, 203109.

21. Rollings, R. C.; Kuan, A. T.; Golovchenko, J. A. Ion Selectivity of Graphene Nanopores. Nat. Commun. 2016, 7, 11408.

22. Heiranian, M.; Farimani, A. B.; Aluru, N. R. Water Desalination with a Single-Layer MoS2 Nanopore. 2015, 6, 8616.

23. Liu, K.; Feng, J.; Kis, A.; Radenovic, A. Atomically Thin Molybdenum Disulfide Nanopores with High Sensitivity for DNA Translocation. ACS Nano 2014, 8, 2504-2511. 
24. Feng, J.; Liu, K.; Graf, M.; Lihter, M.; Bulushev, R. D.; Dumcenco, D.; Alexander, D. T. L.; Krasnozhon, D.; Vuletic, T.; Kis, A.; Radenovic, A. Electrochemical Reaction in Single Layer MoS2: Nanopores Opened Atom by Atom. Nano Lett. 2015, 15, 3431-3438. 